\title{
Could a coffee culture be ruining our health?
}

EDITORIAL

\author{
Moyez Jiwa, Epi Kanjo, Catherine Krejany
}

Melbourne Clinical School, The University of Notre Dame Australia, Werribee, VIC, Australia

To Cite: Jiwa M, Kanjo E, Krejany C. Could a coffee culture be ruining our health? JHD. 2019;4(3):205-209.

https://doi.org/10.21853/JHD.2019.91

\section{Corresponding Author:}

Catherine Krejany

Melbourne Clinical School

The University of Notre Dame Australia

Werribee, VIC, Australia

catherine.krejany@nd.edu.au

\section{Copyright:}

(c) 2019 The Authors. Published by Archetype Health Pty Ltd. This is an open access article under the CC BY-NC-ND 4.0 license.

\section{SUMMARY}

Being overweight is now the norm. It is hypothesised eating between meals is a significant part of the problem. Habits that are part of social intercourse are implicated in obesity insofar as those who opt for caffeinated drinks when socialising between meals are also more likely to concurrently consume high energy-dense snacks. The consumption of sugar as a contributor to obesity is well established and experts also agree that the habit of eating for pleasure rather than hunger is strongly implicated in weight gain. Such habitual eating patterns may offer opportunity for feasible lifestyle change when framed appropriately. A promising strategy involves visualising avatars of a healthier future self as well as identifying the circumstances in which threats to that outcome can be tackled immediately. Though promising, there is limited evidence that such a strategy can be effective yet.

Key words

Obesity, research, nutrition, triggering, mental contrasting

\section{INTRODUCTION}

In most Organisation for Economic Cooperation and Development (OECD) countries there are now more people who are obese or overweight than there are people who are normal weight. Increasing daily total energy intake and decreasing physical activity are contributing to the increase in numbers of overweight individuals. Patterns of eating behaviour are also changing and the traditional three meals a day in Western culture is being supplemented by frequent "between meals" consumption. ${ }^{1,2}$ Recent commentary has focused on the idea that what we eat between meals is the problem. It is proposed that snacking behaviour has increased over time; for example, children in the United States are now trending towards three snacks per day, which contribute more than 27 per cent of their total daily energy intake. ${ }^{3}$ It is important to note that snacking behaviour varies significantly between cultures. ${ }^{1}$ Regular consumption of nutrient-rich healthy snacks can be protective against weight gain. However, many people exhibit eating behaviours where there is frequent consumption of high, energy-dense, low-nutritional quality foods between meals. ${ }^{2,4}$ Identification of these problematic snacking behaviours and the translation of respectful and sustainable strategies to combat them remains the challenge. Those attempting to address unhealthy snacking behaviours are also pitted against an obesogenic environment with an efficient marketing machine that is highly effective in promoting sales of calorific food and drink. In this editorial, we review some of this literature and question whether an effective public health strategy to manage obesity would be to focus on persuading people to avoid eating between meals. 


\section{When is a snack a meal?}

The challenge with framing the issue in this way is the lack of consensus about what constitutes a "snack". 1,5 Snacking is a word that seems to come laden with negative connotations, conjuring up images of eating a muffin with your morning coffee along with many evenings spent on the couch crunching on cheese flavoured chips. Imagine the following eating habits of a full-time clerical worker: breakfast at the desk, a couple of midmorning biscuits, sandwiches and crisps for lunch, and an afternoon treat, with several cups of tea or coffee in between. Is this "snacking" or "grazing"? Some have defined snacks as "food eaten between meals", however, for others eating more than three times a day is normal. ${ }^{2}$ Such people are not necessarily overweight and are dubbed "grazers".

Regular snacking may simply be the way an individual consumes what they require; frequent consumption of healthy foods can have a regulatory effect on total energy intake and contribute to nutritional quality. ${ }^{2}$ But others are consuming snacks that are calorie dense, of poor nutritional value, and high in sugar and fat. These types of "snack" foods are being consumed in habitual eating patterns that are not triggered by hunger and do not initiate satiety post-consumption increasing the likelihood of contributing to weight gain. ${ }^{2,6} \mathrm{We}$ propose that it is this type of snacking behaviour that can be targeted for effective lifestyle change for those individuals motivated and able to make such a change. Therefore, any attempt to tackle this issue needs to take into account what someone is eating throughout the day and not just at times that are unconventional for eating in that culture.

\section{Eating frequency, the food environment, and the role of habit}

Willingness to eat is driven by a combination of lack of satiety and food reward-the anticipated pleasure of eating. ${ }^{6}$ Desire to eat can be moderated somewhat by recent consumption, but we are nearly always ready to eat given the opportunity. Recent data suggest that people are tempted by high energy-dense foods, and food attention for these types of foods seems to be heightened in overweight or obese people. ${ }^{7}$ In today's society where food is available everywhere and around the clock, especially in larger cities, eating more frequently is easily achieved. In recent years, this is compounded by the rise of ubiquitous home delivery services that will deliver whatever is desired to your doorstep with just a few taps on your mobile phone app. Eating when we are not hungry is a key factor in the development of obesity. Several studies have demonstrated that eating often occurs for reasons of habit rather than hunger or boredom; for example, Verhoeven et al reported in the British Journal of Health Psychology:

The present study included the intention to eat more healthily, habit strength and the Power of

Food, and demonstrated that habit strength is the most important predictor of unhealthy snacking behaviour, stressing the importance of addressing habits in further research and interventions concerning unhealthy snacking behaviour. ${ }^{8}$

\section{Food and drink habits}

There is an increasing trend for daily consumption of warm caffeinated drinks, particularly coffee. This is especially problematic where the coffee culture involves concomitant consumption of sugary snacks together 
with coffee prepared with additional milk, cream, and sweeteners. In cultures where this is a relatively new trend, the adverse health implications have been noteworthy. In Japan, for example, habitual coffee but not green tea consumption is inversely associated with metabolic syndrome, a sequalae of obesity. ${ }^{9}$ Similar observations have been made in the US where coffee drinking is a more established norm. ${ }^{10}$ At the same time, it has been noted that those who are more likely to consume coffee and other commercial drinks exhibit other potentially unhealthy habits:

Frequent drinking of commercial beverages was associated with frequent snacking $(P=0.002)$, meal skipping $(P=0.006)$, eating out $(P=0.003)$, eating delivered foods $(P=0.000)$, processed foods $(P$ $=0.001)$, and sweets $(P=0.002)$, and drinking alcoholic beverages $(P=0.029){ }^{11}$

A significant contribution to the discourse on obesity has been a review of the role of sugar. Animal models have demonstrated that when offered the free choice of sweet and fat-rich meals animals will not experience satiety and therefore consume more than they need rather than avoiding food when they would not normally be eating. This model may explain why people who eat sugary treats tend to eat more. ${ }^{12}$ These findings reflect observational studies in humans where, in a Swedish population, obese women snacked more often and had higher energy intake than their leaner peers. ${ }^{13}$ These individuals selected cakes cookies, candies, chocolate, and desserts for snacks more often than did lean controls. Similarly, in a French study, obese women were more likely to be snackers consuming up to $430 \mathrm{Kcal}$ of extraprandial intake daily, with snacking occurring mainly in the afternoon. ${ }^{14}$

\section{Changing norms and the external environment}

There are many other changing trends that contribute to snacking behaviours. Family influences play a large role in the eating patterns of children determining what, when, and how they eat. Findings from recent studies measuring exercise, screen time, snacking, and sleep show that the frequency of eating along with parenting style is to blame for the increase in caloric uptake in Australian children. ${ }^{15,16}$ These studies found positive associations with an increase in consumption of energy-dense foods, lower levels of physical activity, frequent television viewing, and computer use. Children as young as 11 are spending more time in front of screens, and less time on physical activity resulting in poorer body composition. ${ }^{16}$

Another problematic trend is that overweight is now regarded as normal, and studies report that people do not recognise that they are overweight. ${ }^{17}$ Being surrounded by friends and family who are also overweight or obese has a normalising affect and promotes a misperception of a healthy body weight image and a lesser inclination to address the issue. ${ }^{18}$ Much has also been written about our modern obesogenic environment with oversupply of food, lack of physical activity, and the ready availability and marketing of high energy dense foods-all major drivers of the obesity epidemic.

\section{Tackling the issue - Triggering behaviour change}

It is clear then, that obesity is driven by many multifaceted influences, and there cannot be any singular approach to this problem. We can, however, hope for improvements in personal health when identifying 
individuals who are motivated and able to make behavioural change. We propose that targeting snacking frequency and consumption of energy-dense "between meal" snacks is a viable option to raise awareness of the intake of calories excess to requirement. This may "on-board" individuals towards better food choices.

One such approach may be to identify what people are eating and when they are eating, with reference to their normal eating habits. For people whose eating is driven by habits rather than hunger, it may be that those habits can be addressed.

People are not good at recognising when they are triggered to snack. The triggers are varied and may include boredom anxiety, or social or cultural cues. Recent promising approaches to triggering positive behaviour change involve self-regulatory strategies that need to be combined: (1) identifying the circumstances in which one tends to consume unhealthy snacks (eg, chocolate when bored); and (2) presenting a tangible positive future outcome if one were to alter this behaviour (eg, personalised avatar with a different desired body shape). By combining these elements in one intervention it may be possible to reinforce the desired behaviour by associating the negative behaviour as a hindrance to the favoured outcome. The desired outcome is then perceived as feasible: "If I stop eating chocolate when I'm bored (eg, at 3pm), then it is more likely that I will have the body shape I want". The person then forms a strong commitment to realise the desired future by seeing that change may be feasible and anchored to a specific and recurring event. ${ }^{19,20}$ Framing snacking behaviours, such as the sugary snacks we consume with coffee, as a negative influence on a desired personal goal may be a first step in engaging people to address problematic eating habits.

\section{REFERENCES}

1. Hess JM, Jonnalagadda SS, Slavin JL. What is a snack, why do we snack, and how can we choose better snacks? A review of the definitions of snacking, motivations to snack, contributions to dietary intake, and recommendations for improvement. Advances in Nutrition 2016;7(3):466-75.

2. Bellisle F. Meals and snacking, diet quality and energy balance. Physiology $\mathcal{E}$ behavior 2014;134:38-43.

3. Piernas C, Popkin BM. Trends in snacking among US children. Health affairs 2010;29(3):398-404.

4. Murakami K, Livingstone M. Eating frequency in relation to body mass index and waist circumference in British adults. International Journal of Obesity 2014;38(9):1200.

5. Johnson GH, Anderson GH. Snacking definitions: impact on interpretation of the literature and dietary recommendations. Critical reviews in food science and nutrition 2010;50(9):848-71.

6. Rogers PJ, Brunstrom JM. Appetite and energy balancing. Physiology EF Behavior 2016;164:465-71. doi: https://doi.org/10.1016/j.physbeh.2016.03.038

7. Nijs IMT, Muris P, Euser AS, et al. Differences in attention to food and food intake between overweight/obese and normal-weight females under conditions of hunger and satiety. Appetite 2010;54(2):243-54. doi: https://doi.org/10.1016/j.appet.2009.11.004

8. Verhoeven AA, Adriaanse MA, Evers C, et al. The power of habits: Unhealthy snacking behaviour is primarily predicted by habit strength. British Journal of Health Psychology 2012;17(4):758-70.

9. Hino A, Adachi $\mathrm{H}$, Enomoto $\mathrm{M}$, et al. Habitual coffee but not green tea consumption is inversely associated with metabolic syndrome: an epidemiological study in a general Japanese population. Diabetes research and clinical practice 2007;76(3):383-89. 
10. Duffey KJ, Popkin BM. Shifts in patterns and consumption of beverages between 1965 and 2002. Obesity 2007;15(11):2739-47.

11. Kim H, Han SN, Song K, et al. Lifestyle, dietary habits and consumption pattern of male university students according to the frequency of commercial beverage consumptions. Nutrition research and practice 2011;5(2):124-31.

12. la Fleur SE, Luijendijk MC, van der Zwaal EM, et al. The snacking rat as model of human obesity: effects of a free-choice high-fat high-sugar diet on meal patterns. International Journal of Obesity 2014;38(5):643.

13. Forslund HB, Lindroos A, Sjöström L, et al. Meal patterns and obesity in Swedish women-a simple instrument describing usual meal types, frequency and temporal distribution. European journal of clinical nutrition 2002;56(8):740.

14. Basdevant A, Craplet C, Guy-Grand B. Snacking patterns in obese French women. Appetite 1993;21(1):17-23.

15. Vivarini P, Kerr JA, Clifford SA, et al. Food choices: concordance in Australian children aged 11-12 years and their parents. BMJ Open 2019;9(Suppl 3):147. doi: 10.1136/bmjopen-2017-020898

16. Dumuid D, Wake M, Clifford S, et al. The association of the body composition of children with 24-hour activity composition. The Journal of pediatrics 2019;208:43-49. e9.

17. Flanagan R, Waugh D, Meng X, et al. Who knows what 'healthy weight'looks like? The Journal of Health Design 2018;3(2):104-8.

18. Muttarak R. Normalization of plus size and the danger of unseen overweight and obesity in England. Obesity 2018;26(7):1125-9.

19. Adriaanse MA, Oettingen G, Gollwitzer PM, et al. When planning is not enough: Fighting unhealthy snacking habits by mental contrasting with implementation intentions (MCII). European Journal of Social Psychology 2010;40(7):1277-93.

20. Ossolinski G, Jiwa M, McManus A, et al. Do images of a personalised future body shape help with weight loss? A randomised controlled study. Trials 2017;18(1):180. doi: 10.1186/s13063-017-1907-6

\section{ACKNOWLEDGEMENTS}

None

\section{PEER REVIEW}

Not commissioned. Externally peer reviewed.

\section{CONFLICTS OF INTEREST}

The authors declare that they have no competing interests.

\section{FUNDING}

None

\section{ETHICS COMMITTEE APPROVAL}

Not applicable 